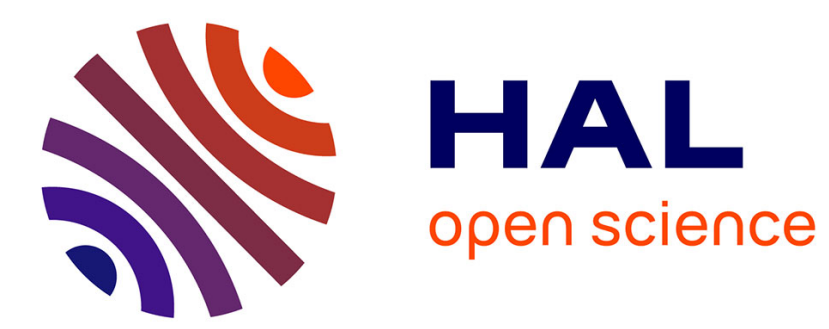

\title{
Electrocristallisation. Théorie et applications
}

\author{
R. Winand
}

\section{- To cite this version:}

R. Winand. Electrocristallisation. Théorie et applications. Journal de Physique IV Proceedings, 1994, 04 (C1), pp.C1-55-C1-73. 10.1051/jp4:1994104 . jpa-00252446

\section{HAL Id: jpa-00252446 https://hal.science/jpa-00252446}

Submitted on 1 Jan 1994

HAL is a multi-disciplinary open access archive for the deposit and dissemination of scientific research documents, whether they are published or not. The documents may come from teaching and research institutions in France or abroad, or from public or private research centers.
L'archive ouverte pluridisciplinaire HAL, est destinée au dépôt et à la diffusion de documents scientifiques de niveau recherche, publiés ou non, émanant des établissements d'enseignement et de recherche français ou étrangers, des laboratoires publics ou privés. 


\title{
Electrocristallisation. Théorie et applications
}

\author{
R. WINAND
}

Université Libre de Bruxelles, Service Métallurgie-Electrochimie, CP 165, 50 avenue F.D. Roosevelt, 1050 Bruxelles, Belgique

\section{ABSTRACT}

After a short summa:y of the theory, Winand's diagram is introduced. This diagram gives the fields of stability of Fischer's main types of electrodeposits as a function of two main parameters : the ratio of the current density to the diffusion limiting current density (mass transfer) and the inhibition.

The diagram has been used at Université Libre de Bruxelles as the basis for the chloride electrodeposition of metals, for high current density plating and electrorefining, and for initiating research concerning organic additives. Important industrial applications have been derived, especially in continuous steel sheet electroplating. New research areas in nucleation, and in the growth of thin films and foils are discussed.

\section{INTRODUCTION}

L'électrocristallisation est envisagée dans ce texte comme la science et la technologie d'obtention d'un dépôt solide de métal à la cathode d'une cellule d'électrolyse. De plus, seul le cas des solutions aqueuses est pris en considération.

Un grand nombre de paramètres influencent le type de dépôt : la densité de courant $\mathrm{J}$; la concentration en ion métallique à décharger $\mathrm{C}_{\mathrm{Me}^{2+}}$; l'agitation; la température; le $\mathrm{pH}$; la présence d'autres cations et anions; la complexation; les inhibiteurs; le substrat. Une idée guide est dès lors nécessaire pour établir des programmes expérimentaux permettant d'atteindre des objectifs donnés dans un temps raisonnable. Par ailleurs, alors que la recherche fondamentale fait appel à des monocristaux, les dépôts industriels sont largement polycristallins. Il faut donc établir un pont entre les deux approches. 


\section{THIEORIE DE L'ELECTROCRISTALLISATION [1-4]}

11 n'existe pas de théorie de l'électrocristallisation qui permette, au départ de grandeurs ne faisant appel à aucun paramètre ajustable, de prévoir la structure d'un dépôt électrolytique. La "théorie" est donc largement basée sur l'expérimentation, et est établie par comparaison avec la cristallisation physique.

Dans ce dernier cas, les cristaux métalliques sont formés par solidification d'un métal liquide ou par condensation d'une vapeur métallique, en l'absence d'atomes étrangers et sans transfert de charge. En l'absence de tout cristal initial du métal, une nucléation tridimensionnelle est nécessaire. Toute augmentation de la sursaturation augmente la fréquence de germination et diminue le diamètre des germes. Un facteur important, souvent perdu de vue, est la tension interfaciale qui intervient au cube dans l'expression du travail de germination alors que la sursaturation n'y figure qu'au carré. Les expériences de Volmer et Estermann ont montré que les atomes venant de la phase fluide ne sont pas incorporés dans le réseau à l'endroit de la première collision, en sorte que la diffusion superficielle d'atomes adsorbés joue un rôle important dans la croissance des cristaux, laquelle fait aussi intervenir les concepts de germination bidimensionnelle (nouveau plan en surface du cristal) et monodimensionnelle (nouvelle rangée). Jusqu'à ce qu'il ait été démontré que la croissance des cristaux peut se faire en spirale au départ d'une dislocation vis, les idées de Kossel et Stranski ont prévalu : la croissance des cristaux a lieu en couches monoatomiques répétées, après germination bidimensionnelle, le processus d'incorporation des atomes dans le réseau ayant lieu en un site privilégié répétitif (kink site), situé à l'avant d'une rangée qui progresse le long d'une marche de croissance. Il y a ainsi compétition entre la croissance verticale, liée à la fréquence de germination bidimensionnelle, et la croissance horizontale, liée à la vitesse d'incorporation des atomes au "kink site". Un cristal parfait croissant dans des conditions proches de l'équilibre sera délimité par les plans à la surface desquels l'énergie d'incorporation d'un atome est la plus petite, car ce sont ceux qui ont le temps de se développer latéralement.

En augmentant la sursaturation, on rend possible l'apparition de nouveaux plans, alors que les précédents ne sont pas encore complets et la forme extérieure du cristal en est modifiée. Par exemple, si la forme de presque équilibre d'un cristal cubique à faces centrées est basée sur des pyramides tétragonales, on passe à des formes cubiques ou rhombododécaédriques en augmentant la sursaturation. Audelà, la forme s'arrondit et on observe des dendrites. Finalement, de nouveaux cristaux apparaissent.

En électrocristallisation, le problème se complique du fait de la présence de molécules, atomes et ions adsorbés à l'interface où a lieu un transfert de charge au départ d'un ion plus ou moins solvaté sous l'effet d'un champ électrique de l'ordre de $10^{7} \mathrm{~V} \mathrm{~cm} \mathrm{~cm}^{-1}$. On cherche donc plutôt à faire usage ici des connaissances accumulées en cristallisation physique, en cherchant une corrélation entre les formes cristallines observées et un paramètre physique mesurable, caractéristique de la sursaturation, en l'occurrence la surtension électrochimique. Malheureusement, il s'agit là d'un paramètre complexe qui peut être séparé en quatre termes additifs [5] : les surtensions de transfert de charge, de diffusion, de réaction et de cristallisation. La surtension globale seule ne permet pas d'obtenir la corrélation recherchée. La surtension de cristallisation ne peut être obtenue que 
par différence dans la plupart des cas, après une analyse détaillée de la surtension globale, ce qui nécessite des recherches expérimentales longues et coûteuses.

L'auteur a dès lors suggéré $[6,7]$ qu'il soit fait usage de deux paramètres principaux permettant de délimiter dans un plan des zones de prédominance de structures cristallines déterminées : le rapport $\mathrm{J} / \mathrm{C}_{\mathrm{Me}}{ }^{\mathrm{z}+}$ de la densité de courant à la concentration en ions métalliques à décharger (ce facteur caractérise le transfert de masse à l'électrode; si les conditions hydrodynamiques sont variables, il est préférable d'employer le rapport $\mathrm{J} / \mathrm{J}_{\mathrm{d}}$ où $\mathrm{J}_{\mathrm{d}}$ est la densité de courant limite de diffusion) et l'intensité de l'inhibition. Bien que l'approche initiale ait été largement intuitive, on peut montrer $[2,8]$ que si les conditions hydrodynamiques à l'électrode sont connues et en l'absence d'additifs organiques, on peut attribuer une valeur de la surtension de diffusion et de la surtension de transfert de charge en tout point d'un diagramme qui comporte les deux parametres susmentionnés comme coordonnées.

Si le transfert de masse est en général bien compris, l'inhibition mérite quelques mots d'explication. D'après Fischer [4, 9], l'inhibition est due à la présence à la surface de l'électrode (dans la double-couche ou dans la couche de diffusion) de substances (molécules, atomes ou ions) différentes de $\mathrm{Me}^{z+}$ et de l'adatome correspondant. Ces substances freinent le processus cathodique et sont appelées inhibiteurs. Elles ne couvrent en général pas toute la surface cathodique et ménagent des sites actifs. Elles sont adsorbées physiquement ou chimiquement. Elles ont un effet sur le processus cathodique et donc sur les quatre types de surtensions, ainsi que sur la structure métallographique et la texture d'orientation d'ensemble des dépôts. La sensibilité d'un métal aux inhibiteurs est d'autant plus élevée que l'affinité de la surface pour cet inhibiteur est élevée et que le coefficient de diffusion superficielle des adatomes est faible.

On distingue ainsi des métaux normaux ( $\mathrm{Cd}, \mathrm{Zn}, \mathrm{Sn}, \mathrm{Ag})$, peu sensibles aux inhibiteurs, caractérisés par une basse température de fusion et une densité de courant d'échange Jo élevée; des métaux inertes ( $\mathrm{Fe}, \mathrm{Ni}, \mathrm{Co}, \mathrm{Pt}, \mathrm{Cr}, \mathrm{Mn}$ ), très sensibles aux inhibiteurs, à haute température de fusion et à $\mathrm{J}_{0}$ très petit; des métaux intermédiaires $(\mathrm{Au}, \mathrm{Cu}, \mathrm{Ag})$. . La nature des inhibiteurs est également à prendre en considération : les cations inorganiques non déchargeables à la cathode n'ont qu'une faible influence, sauf pour le $\mathrm{pH}$ qui intervient dans l'inhibition secondaire : certains anions inorganiques sont activants $\left(\mathrm{Cl}^{-}, \mathrm{Br}^{-}, \mathrm{I}^{-}\right)$, d'autres sont

inhibants $\left(\mathrm{BF}_{4}^{-}, \mathrm{NH}_{2} \mathrm{SO}_{2}^{2-}, \mathrm{ClO}_{4}^{-}\right)$, ou intermédiaires $\left(\mathrm{NO}_{3}^{-}, \mathrm{SO}_{4}^{\overline{4}}\right)$; les substances organiques en solution sont très inhibantes si elles sont adsorbées sur le métal et n'ont pas d'affinité pour l'eau; les substances organiques en suspension colloïdale sont souvent fortement adsorbées et provoquent l'apparition de l'inhibition secondaire due à des changements locaux de $\mathrm{pH}$ induisant des précipitations superficielles.

L'intensité de l'inhibition est donc un paramètre complexe. Il sera néanmoins considéré ici comme lié de manière biunivoque à la concentration d'une espèce en solution ou adsorbée sur l'électrode, espèce appelée l'inhibiteur.

D'une manière générale, l'expérimentation conduit à des constatations fort proches de la cristallisation physique [10-14]. On constate cependant que les dislocations vis sont très aisément bloquées par des adsorbats, en sorte que le 
mécanisme de croissance par germination bi-et monodimensionnelle avec site d'incorporation répétitif joue un rôle prépondérant [15-29]. Les marches de croissance sont en général d'épaisseur largement supérieure à la monocouche atomique (bunching effect) [30-32]. Les formes cristallines obtenues résultent donc d'une compétition entre la croissance latérale parallèle au substrat et la croissance perpendiculaire à celui-ci. Cette dernière dépend du reste beaucoup de la fréquence de germination bidimensionnelle. On a observé que l'épaisseur des couches de croissance augmente avec la densité de courant, mais diminue lorsque l'inhibition augmente. Il en résulte que la vitesse latérale de croissance diminue à inhibition constante quand la densité de courant augmente, ce qui favorise l'obtention de cristaux isolés, voire de dendrites. Par contre, lorsque l'inhibition augmente à densité de courant constante, la vitesse latérale de croissance augmente, ce qui favorise l'obtention de dépôts denses. A très faible inhibition et à très faible valeur du rapport $\mathrm{J} / \mathrm{CMe}_{\mathrm{M}}{ }^{\mathrm{z}}$ il est possible que la surtension soit insuffisante pour provoquer la germination bidimensionnelle. La croissance du dépôt ne peut alors avoir lieu que s'il y a des dislocations vis actives. Enfin, lorsque l'intensité de l'inhibition et/ou la valeur du rapport $\mathrm{J} / \mathrm{C}_{\mathrm{Me}{ }^{\mathrm{z}}}$ devien(nen)t suffisante(s), de nouveaux cristaux sont générés dans l'épaisseur du dépôt par germination tridimensionnelle. Toutes ces considérations sont résumées dans la figure 1.

Fischer [4] a proposé de caractériser les dépôts électrolytiques polycristallins en se basant sur cinq types principaux de structure de coupe métallographique :

à cristaux isolés orientés dans le champ (FI)

à reproduction de la base (BR)

à cristaux maclés $(Z)$

à texture orientée dans le champ (FT)

à structure non orientée, dispersée (UD)

Ces types principaux sont repris dans la figure 1, où l'on voit ainsi apparaître leurs domaines de stabilité. La figure 2 n'est qu'une représentation simplifiée de la figure 1. Il faut évidemment garder à l'esprit que les frontières entre les types de dépôt ne sont pas marquées de manière abrupte, sauf le passage de la germination bidimensionnelle à la germination tridimensionnelle. En outre, ce diagramme ne tient pas compte de I'influence du substrat : il est basé uniquement sur les paramètres de l'électrolyse.

Il est à noter que Fischer a introduit en outre les dépôts nodulaires (N) et rythmiques lamellaires (RL) qui correspondent à des cas particuliers qu'il est difficile d'inclure d'une maniere générale dans les figures 1 et $2[33,34]$.

\section{AIPILICATIONS}

En vue d'illustrer comment les diagrammes des figures 1 et 2 peuvent être utilisés, quelques résultats expérimentaux obtenus dans les laboratoires de l'auteur sont à présent discutés et leur emploi industriel mis en évidence. La première idée a été de valider le diagramme. Au cours de ce processus, des applications industrielles ont déjà pu être générées. Comme il est apparu très tôt que les additifs organiques ont un comportement complexe, les solutions pures ont d'abord été étudiées. La coordonnée verticale a, dans ce cas, été parcourue en changeant de 
métal ou d'anion. La coordonnée horizontale a été analysée en changeant la densité de courant et l'agitation. Les additifs organiques sont traités séparément.

\section{Validation du diagramme en l'absence d'additifs organique}

\section{La coordonnée verticale: intensité de l'inhibition}

En l'absence d'additif organique, on peut modifier l'intensité de l'inhibition en changeant de métal ou d'anion.

L'argent, déposé au départ de solutions de nitrates [35], présente une densité de courant d'échange très élevée (10 à $100 \mathrm{~A} \mathrm{dm}^{-2}$ ). Il faut donc s'attendre à se trouver dans les figures 1 et 2 le long d'une ligne horizontale fort proche de l'axe des abscisses.

C'est effectivement le cas : les résultats expérimentaux montrent qu'après une mince couche de cristaux de type BR croissant par épitaxie sur le substrat en argent, la structure évolue vers des cristaux dendritiques de type FI, ce qui est bien connu dans l'électroraffinage industriel de l'argent (fig. 3).

Le cuivre déposé au départ de solutions de sulfates [26, 36, 37] présente une densité de courant d'échange moins élevée $\left(0,2 \mathrm{à} 2 \mathrm{~A} \mathrm{dm}^{-2}\right)$. Les dépôts devraient donc se trouver dans les figures 1 et 2 le long de lignes horizontales à faible ou moyenne inhibition. On trouve en effet expérimentalement des structures BR (Z), FT et UD lorsque le rapport $\mathrm{J} / \mathrm{C}_{\mathrm{Me}} \mathrm{z}^{\mathrm{t}}$ augmente. Par contre, en solutions concentrées de chlorures $[38,39]$, la densité de courant d'échange pour la réduction

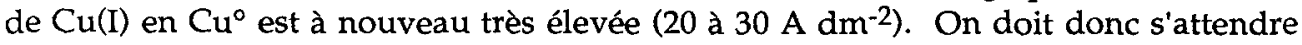
à un comportement similaire à celui de l'argent en solutions de nitrates, ce qui est effectivement constaté expérimentalement (fig. 4).

Le cobalt déposé en solutions de sulfates présente des densités de courant d'échange très basses $\left(10^{-8}\right.$ à $\left.10^{-10} \mathrm{~A} \mathrm{dm}^{-2}\right)$ et conduit à des structures métallographiques très dégradées de type FT et UD, souvent $N$, caractéristiques d'une forte inhibition. Par contre, en remplaçant les ions sulfates par des ions chlorures $[40,41]$, les densités de courant d'échange augmentent considérablement : $10^{-5} \mathrm{~A} \mathrm{dm}^{-2}$ si le pH est inférieur à 3,5, ce qui donne encore des structures UD d'inhibition forte, et $10^{-2} \mathrm{~A} \mathrm{dm}^{-2}$ si le pH est compris entre 4 et 4,5 , ce qui conduit a des structures BR et FT qui correspondent à une inhibition moyenne (fig. 5). Une observation intéressante a été faite : il est possible d'obtenir en solutions de chlorure à $\mathrm{pH}$ moyen $(3-3,5)$ des dépôts brillants sans faire usage d'inhibiteurs (fig. 6), ce résultat pouvant être interprété sur la base d'une inhibition secondaire due à des espèces adsorbées (42). Des applications industrielles devraient pouvoir en découler.

Sur la base des quelques exemples décrits ci-dessus, on peut déduire que le comportement d'un métal dans une solution exempte d'additif organique peut être représenté de manière approximative par des horizontales dans les figures 1 et 2 dont la position le long de l'axe vertical dépend de la densité de courant d'échange $J_{0}$ : plus la valeur de $J_{0}$ est élevée, plus faible est l'inhibition. 


\section{La coordonnée horizontale : le transfert de masse}

La validité de cette coordonnée a été vérifiée dans le cas du cuivre en solution de sulfates, en faisant usage d'une cellule à circulation à canal de section rectangulaire [36]. Il a été constaté que la transition de FT à UD (assez aisée à observer puisque UD implique l'apparition de nouveaux cristaux dans l'épaisseur $\mathrm{du}$ dépôt par germination $3 \mathrm{D}$ ) se produit à un rapport $\mathrm{J} / \mathrm{J}_{\mathrm{d}}$ proche de 0,35 , indépendamment de la vitesse d'écoulement. Evidemment, comme $\mathrm{H}_{2} \mathrm{SO}_{4}$ n'est qu'un faible inhibiteur, la rugosité de surface augmente avec l'épaisseur de dépôt conformément à la théorie de Despic [43].

En conséquence, des dépôts de cuivre denses ayant une rugosité de surface acceptable pour l'électroraffinage ont pu être obtenus en laboratoire jusqu'à des épaisseurs de $2 \mathrm{~mm}$ à des densités de courant de $30 \mathrm{~A} \mathrm{dm}^{-2}$, avec une vitesse d'écoulement d'électrolyte parallèle aux électrodes de $3 \mathrm{~m} \mathrm{~s}^{-1}$. Au-delà de cette épaisseur, des additifs organiques sont indispensables [44]. Par contre, en galvanoplastie, des densités de courant bien supérieures à $50 \mathrm{~A} \mathrm{dm}^{-2}$ peuvent être envisagées [36]. Toutefois, les débouchées industriels principaux dans ce domaine ont trait au dépôt de zinc sur acier. A l'échelle du laboratoire, faisant toujours usage de cellule à circulation à canal de section rectangulaire, avec une solution à $80 \mathrm{~g} / 1 \mathrm{Zn}+2$ et $135 \mathrm{~g} / 1 \mathrm{H}_{2} \mathrm{SO}_{4}$ sans additif, à $50^{\circ} \mathrm{C}$ avec anode soluble en zinc et cathode en tôle d'acier doux, il a été montré $[45,46]$ qu'on obtient des dépôts de très bonne qualité à des densités de courant très élevées (200 à $300 \mathrm{~A} \mathrm{dm}^{-2}$ ) (fig. 7). Les recherches, poursuivies en vue de permettre le revêtement électrolytique en continu de tôles d'acier au défilé à la grande échelle du laboratoire, puis à l'échelle pilote et enfin en accompagnant l'implantation en exploitation industrielle $[47,48]$ a conduit à la mise au point de procédés et d'équipements nouveaux (ULB-Groupe Cockerill Sambre - BELT CELL jusqu'à $300 \mathrm{~A} \mathrm{dm}^{-2}$; FLASH CELL jusqu'à $450 \mathrm{~A}$

$\mathrm{dm}^{-2}$ ). Des alliages de zinc ont aussi été étudiés à cette occasion [49-51]. On a montré que lorsque le rendement de courant est faible, le dégagement d'hydrogène contribue de manière importante au transfert de masse à la cathode en sorte que pour obtenir des compositions d'alliages homogènes sur toute la largeur de la bande, il est essentiel que les conditions hydrodynamiques et la densité de courant soient constantes sur toute la surface exposée.

\section{Electrocristallisation en présence d'additifs organiques}

Pour l'argent en solutions de nitrates, étant donné la valeur élevée de la densité de courant d'échange, la sensibilité aux inhibiteurs est très faible [52, 53]. C'est ainsi qu'avec la thiourée, il faut atteindre $100 \mathrm{mg} / 1$ (la limite de solubilité) pour convertir soudain la structure BR en UD avec toutefois une contamination élevée en soufre. De meilleurs résultats sont obtenus avec du bitartrate de sodium et de potassium, sans toutefois permettre une exploitation industrielle. Des difficultés analogues sont rencontrées pour améliorer la structure des dépôts de cuivre au départ de solutions de chlorures : il faut $0,3 \mathrm{~g} / 1$ de gélatine pour obtenir des dépôts denses, de type FT-UD [54].

De meilleurs résultats sont obtenus pour les dépôts de cuivre obtenus en solutions de sulfates. En électroextraction du cuivre, il a été montré que des 
polyacrylamides cationiques donnent des dépôts FT avec des concentrations aussi faibles que $1 \mathrm{mg} / \mathrm{l}$ ([55]. De plus, en simulant en laboratoire une partie d'une installation industrielle, il a été constaté qu'en augmentant la concentration en Jaguar (nom commercial d'une gélatine), une ligne "verticale" est suivie dans les figures 1 et 2, conduisant successivement à des structures BR, FT (fig. 8) et finalement UD [56].

Lors du développement d'un nouveau procédé d'électroraffinage du cuivre à haute densité de courant avec Métallurgie Hoboken-Overpelt [44], il s'est avéré nécessaire de faire usage d'additifs organiques pour obtenir les épaisseurs de dépôt requises pour l'exploitation industrielle. L'expérimentation a été faite à la grande échelle pilote de laboratoire, dans un circuit fermé comprenant une cellule à circulation à double canal, du type pseudo-bipolaire à deux anodes et deux cathodes. Il a été constaté que des concentrations très faibles en additifs sont suffisantes, ce qui s'explique si l'on admet que les additifs sont consommés à la cathode à leur propre densitê de courant limite de diffusion, selon les hypothèses de Bockris et Razumney [57]. Une stratégie expérimentale particulière a permis de trouver la combinaison adéquate des trois additifs usuels (ions chlore ou brome, thiourée et gélatine). Dans le cas particulier étudié, dans une solution industrielle contenant $39,6 \mathrm{~g} / 1 \mathrm{Cu}^{2+}$ et $174 \mathrm{~g} / 1 \mathrm{H}_{2} \mathrm{SO}_{4}$, à $20 \mathrm{~A} \mathrm{dm}^{-2}$ avec une vitesse de passage d'électrolyte de 1,4 à $1,8 \mathrm{~m} \mathrm{~s}^{-1}$, un optimum est obtenu à $40 \mathrm{mg} / 1 \mathrm{Br}^{-}+\mathrm{Cl}^{-}$, dont $\mathrm{Br}^{-} 15$ à $25 \mathrm{mgl}$; $0,2 \mathrm{mg} / 1$ de thiourée et $0,1 \mathrm{mg} / 1$ de gélatine ASF. Dans ces conditions, des dépôts de qualité "électroraffinê" sont obtenus pour des épaisseurs allant jusqu'à $12 \mathrm{~mm}$.

Parallèlement, des recherches plus fondamentales ont été faites sur l'influence des additifs sur les dépôts de cuivre obtenus dans des conditions normales d'électroraffinage. Il a été constaté que la gélatine employée seule donne des dépôts FT, c'est-à-dire obtenus en régime de germination bidimensionnelle, jusqu'à des concentrations élevées alors que la surtension globale est élevée dès les faibles concentrations. Par contre, la cystéine, qui possède des propriétés proches de la thiourée, donne des dépôts UD, c'est-à-dire nécessitant la germination tridimensionnelle, dès les faibles concentrations, alors que la surtension est plus faible que celle obtenue avec la gélatine $[58,59]$. Ceci ne peut être expliqué que si la tension interfaciale est prise en considération. Malheureusement, il n'existe pas encore de méthode expérimentale satisfaisante permettant de la mesurer pendant la croissance d'un dépôt. D'autres travaux ont montré qu'il est absolument nécessaire d'ajouter les additifs organiques en continu au lieu de les introduire en une fois au début de l'électrolyse [60]. En effet, les additifs ont souvent une chimie et une électrochimie complexes, impliquant des réactions en solution et aux électrodes, avec consommation des additifs et génération de produits de dégradation qui ont eux-mêmes un comportement complexe et qui peuvent aussi jouer le rôle d'inhibiteurs. Il faut donc simuler aussi bien que possible l'installation industrielle pour obtenir des résultats comparables [61,62].

Ces idées ont été utilisées en vue de mettre au point un capteur permettant le contrôle automatique des additifs en électroraffinage du cuivre [63]. Le montage expérimental, assez complexe, simule l'installation industrielle en conservant le rapport du volume d'électrolyte à la surface cathodique, le rapport du débit de la saignée à la surface cathodique, les conditions hydrodynamiques, le temps de séjour de l'électrolyte dans la cellule d'électrolyse, la densité de courant, l'électrolyte industriel et l'anode soluble. Quant au capteur, il travaille dans des conditions hydrodynamiques bien définies, à des valeurs du rapport $\mathrm{J} / \mathrm{J}_{\mathrm{d}}$ analogues à celles de 
la cellule d'électrolyse industrielle, et permet la mesure en continu d'une surtension cathodique stationnaire sur une bande de cuivre au défilé.

La stratégie de contrôle maintient la concentration en ion chlore et le rapport thiourée/gélatine constants, en sorte que la surtension mesurée ne dépend que d'un seul facteur : le débit global d'additifs organiques ajoutés à la solution. Malgré toutes ces précautions, il s'est avéré nécessaire de stabiliser le potentiel redox de la solution à une valeur proche de celle rencontrée en industrie et d'activer la surface de la bande de cuivre par une courte phase de dissolution anodique. Enfin, ce n'est qu'après que l'épaisseur du dépôt réalisé sur la bande de cuivre du capteur a atteint 30 micromètres que les surtensions atteignent des valeurs comparables à celles mesurées sur les cathodes industrielles. Ceci suggère que, même si les dépôts démarrent en épitaxe sur la bande de cuivre activée, la structure du dépôf est initialement fortement influencée par le substrat et n'atteint celle imposée par l'électrolyse qu'après l'épaisseur susmentionnée. Ceci a en effet été observé récemment au laboratoire en électrodéposant du cuivre sur du titane anodisé sans additif organique [64]. On observe au microscope électronique à transmission une zone très perturbée comportant de nombreuses macles et dislocations, et qui recristallise du reste lentement à température ambiante. Les recherches se poursuivent actuellement en vue de mettre en évidence le rôle de chaque additif autour du point de fonctionnement industriel.

\section{NOUVEAUX DOMAINIES DE RECIHIERCIHIE IEN ELIECIROCRISTALIISATION}

Dans le cadre d'une collaboration avec l'Université du Missouri à Rolla (Prof. Tom O'Keefe), la germination du cuivre sur titane anodisé a été étudiée [65, 66]. Il a été constaté que le film d'oxyde joue un rôle très important pendant la germination : il est possible de diminuer le diamètre des cristaux et d'augmenter leur densité superficielle en augmentant l'épaisseur du film anodique. Sur cette base, la production de feuilles minces de cuivre à usage électronique a pu être développée sur des substrats de titane à oxyde microporeux, dans des électrolytes exempts d'additifs : chaque pore semble jouer le rôle d'un site de nucléation, ce qui donne une très haute densité superficielle de germes. En outre, la séparation de la feuille mince de son substrat est aisée [67].

Enfin, récemment, Vanden Brande [68,69] a montré que, dans des conditions galvanostatiques, pendant le tout début de l'électrodéposition du cuivre sur des substrats en carbone amorphe et en argent polycristallin (obtenus sous vide), on observe un mode de croissance par îlots dont la densité est plus élevée sur argent que sur carbone. La dissolution de certains de ces îlots est observée ensuite, et des zones d'exclusion apparaissent autour des îlots restant, lesquelles continuent alors de crôtre (fig. 9). Il est probable que la plupart des résultats publiés dans la littérature, y compris pour les essais potentiostatiques, négligent les phénomènes observés tout au début de l'électrodéposition, en sorte qu'ils décrivent plus un mode de croissance d'îlots déjà stabilisés plutôt que ceux dus à la germination initiale. Les observations faites dans les conditions galvanostatiques mentionnées plus haut $[68,69]$ montrent que le rendement de courant passe par un minimum lorsque les îlots ont finis de se dissoudre. 
La valeur du rendement de courant de ce minimum augmente avec la densité de courant. Il en est de même de la densité des îlots à ce moment. Il semble possible d'obtenir une couverture complète de la surface par de petits îlots correspondant aux germes initiaux, sans dissolution partielle et donc sans zone d'exclusion, mais cela exige des densités de courant très élevées, incompatibles avec le matériel de laboratoire utilisé dans cette recherche. Il faut noter toutefois que les essais effectués avec les montages de recherche-développement mentionnés plus haut pour la galvanoplastie du zinc sur tôle d'acier au défilé donnent des dépôts complètement couvrant dès le dixième de micromètre, à $450 \mathrm{~A} \mathrm{dm}^{-2}$.

\section{CONCLUSIONS}

Bien qu'il n'existe pas à proprement parler de theorie de I'électrocristallisation, une approche pragmatique basée sur des considérations théoriques et des résultats expérimentaux mentionnés dans la littérature ont permis à l'auteur d'établir un diagramme (figures 1 et 2) qui montre les domaines d'existence de types principaux de dépôts selon la classification de Fischer, en fonction de deux paramètres : $\mathrm{J} / \mathrm{C}_{\mathrm{Me}^{\mathrm{z}}}\left(\mathrm{ou} \mathrm{J} / \mathrm{J}_{\mathrm{d}}\right.$ ) pour le transfert de masse, et l'intensité de l'inhibition.

Jusqu'à présent, ce diagramme est largement qualificatif et des valeurs ne peuvent être attribuées aux coordonnées que pour chaque cas spécifique, en fonction de la nature du métal, du type de solution et de l'inhibiteur organique éventuel. Le diagramme n'est pas applicable que pour les structures imposées par les facteurs de l'électrolyse et non par le substrat.

Dans ces conditions, on peut dire que, lorsqu'il n'est pas fait usage d'additif organique, les types de dépôts observés se trouvent le long d'une horizontale dans le diagramme, en fonction de $\int / \mathrm{CMe}^{z+}$ à vitesse d'écoulement de l'électrolyte constante, la position de cette horizontale le long de l'axe vertical dépendant de la densité de courant d'échange Jo du métal dans la solution étudiée : plus Jo est élevée, plus faible est l'intensité de l'inhibition. De plus, il a été montré que pour le cuivre en solutions de sulfates, la transition de FT à UD a lieu pour une valeur du rapport $\mathrm{J} / \mathrm{J}_{\mathrm{d}}$ pratiquement indépendante de la vitesse de l'écoulement. Quand un additif organique est ajouté à une solution, si des verticales ont pu être observées dans quelques cas, dans d'autres de nombreuses questions restent sans réponse.

Malgré ses limites, le diagramme s'est avéré être un guide utile lorsqu'on cherche à améliorer la structure d'un dépôt cathodique. Notamment des procédés à haute densité de courant en solutions de sulfates, et d'autres en solutions de chlorures, ont pu être mis au point dans les laboratoires de l'auteur. Des améliorations du diagramme peuvent être obtenues en tenant compte du facteur temps (germination, début de la croissance et transition des premières couches influencées par le substrat vers le dépôt dépendant des facteurs de l'électrolyse) et en introduisant de nouveaux logiciels d'analyse d'image permettant de quantifier les structures. 


\section{References}

1 WINAND, R.,

Electrochimie, Notes de cours, Texte et Figures, Presses Universitaires de Bruxelles, Bruxelles, $1973,416 \mathrm{pp}$. and $90 \mathrm{pp}$.

2 WINAND, $\mathrm{R}$.

Electroctrystallization, in : WARREN I.H. (Ed.), Application of Polarization Measurements in the Control of Metal Deposition, Process Metallurgy, Elsevier, Amsterdam, 1984, pp. 47-69.

3 WINAND, R.,

Electrocrystallization - Theory and Applications - Hydromet. 29 (1992) 567-598

4 FISCHER, $\mathrm{H}$.,

Elektrolytische Abscheidung und Elektrokristallisation von Metallen Berlin - Springer

Verlag 1954, p 729.

5 VETTER, K.J.,

Elektrochemische Kinetik, Springer Verlag Berlin, 1961, 698 pp.Electrochemical Kinetics

Theoretical aspects- Translation Eds. : BRUCKENSTEIN, S., and HOWARD, B.,

Academic Press, New York, 1967.

6 WINAND, R.,

Contribution à l'étude de l'électrodéposition en sels fondus et des phénomènes connexes -

Application au cas particulier du zirconium - PhD Thesis, Université Libre de Bruxelles, Bruxelles, 1960.

7 WINAND, R.,

Contribution à l'étude de l'électrodéposition en sels fondus et des phénomènes connexes -

Application au cas particulier du zirconium - Mem. Scient. - Revue Metall., 58 (1961) 25-35.

8 WINAND, R.,

Electrocrystallization : fundamental considerations and application to

high current density continuous steel sheet plating, J. Appl. Electrochem., 21 (1991) 377-385.

9 FISCHER, H.,

Wirkungen der Inhibitoren bei der Elektrokristallisation, Electrochim. Acta, 2 (1960) 50-96.

10 KAISCHEW, R., and MUTAFTSCHIEW, B.,

Elektrolytische Keimbildung und Kugelförmigen $\mathrm{Pt}$ Einkristallelektroden, $\mathrm{Z}$.

Phys. Chem., 204 (1955) 334-347.

11 EICHKORN, G., SCHLITTER, F.W., and FISCHER, H.,

Zum Nachweis der Bildung dreidimensionaler Keime bei der inhibierten

Elektrokristallisation, Z. Phys. Chem., 62 (1968) 1-8.

12 FISCHER, $\mathrm{H}$.,

Electrocrystallization of metals under ideal and real conditions, Angew. Chem. Engl. 8 (1969)

108-19.

13 FISCHER, $\mathrm{H}$.,

The nucleation dependent growth layer - A structure element in electrocrystallization,

Plating, 56 (1969) 1229-33.

14 KLAPKA, V.,

Elektrokrystallisation von Zinn auf Platin unter galvanostatischen Bedingungen

Coll. Czech. Chem. Comm., 34 (1969) 1131-40.

15 FISCHER, $H$.,

Zusammenhänge zwischen Form und Abscheidungsbedingungen elektrolytisch gewachsener Metallkristalle, IV Fremdstroffeinfluss bei der Entstehung von Blockstrukturen im polykristallin Niederschlag, Z. Elektrochem., 54 (1950) 459-77.

16 BACKX, A.,

Over de elektrokristallisatie van koper,

Ph. D Thesis - University of Louvain, (1965).

17 STEINBERG, M.A.,

Growth spirals originating from screw dislocations on electrolytically produced titanium crystals, Nature, 170 (1952) 1119-20. 
18 PICK, H.J.,

Growth spirals in electrodeposited copper, Nature, 176 (1955) 693.

19 SEITER, H., FISCHER, H., and ALBERT, H.,

Spiral wachstum bei der Elektrokristallisation von Kupfer,Naturwissensch., 45 (1958) 127.

20 KHOLSCHUTTER, V., and TORRICELLI, A.,

Uber elektrolytische Kristallisationsvorgänge, IV. Versuche über Einzelkristalbildung,

Z. Elektrochem., 38 (1932) 213-27.

21 BARNES, S.C.

Cathode polarization and the growth habit of electrodeposited copper, Electrochim. Acta, 3 (1961) 79-86.

22 WRANGLEN, G., Dendrites and growth layers in the electrocrystallization of metals, Trans. R. Inst. Technol. Stockholm, $\mathrm{n}^{\circ} 94$ (1955) 41.

23 PICK, H.J., STOREY, G.G., and VAUGHAN, T.B., The structure of electrodeposited copper - I. An experimental study of the growth of copper during electrodeposition, Electrochim. Acta, 2 (1960) pp 165-78.

24 BARNES, S.C., STOREY, G.G., and PICK, H.J., The structure of electrodeposited copper - III. The effect of current density and temperature on growth habit, ibid., 2 (1960) 195-206.

25 BUDEVSKI, E., On the mechanism of electrocrystallization, J. Crystal Growth, 13/14 (1972) 93-6.

26 WINAND, R., Electrocrystallization of copper, Trans. Sect. C. IMM, 84 (1975) C 67-75.

27 POSTL, D., EICHKORN, G, and FISCHER, H., Zur thermodynamik und Kinetic von Kristallisationsvorgängen bei der Elektrokristallisation von Kupfer, Z. Phys. Chem., 77 (1972) 138-4.

28 EICHKORN, G. and FISCHER, H., Kristallisationsüberpannung der inhibierten Elektrokristallisation bei Keimbildung und Wachstum von Wachstumsschichten, Z. Phys. Chem., 53 (1967) 29-51.

29 FISCHER, H., Electrocrystallization of metals under ideal and real conditions, Angew. Chem. Engl., 8 (1969) 108-19.

30 FRANK, F.C., On the kinematic theory of crystal growth and dissolution processes, in : DOREMUS, R.H., ROBERTS, B.W., and TURNBULL, D., (Eds.), Growth and Perfection of Crystals, Chapman and Hall, London, 1958 pp. 411-418.

31 CABRERA, K., and VERMILEYA, D.A., The growth of crystals from solution, ibid. pp. 393-408.

32 EICHKORN, G., and FISCHER, H., Uber die Bildung zweidimensionaler Netzebenenkeime der Gleichgewichstsform bei der inhibierten Elektrokristallisation - Z. Phys. Chem., 61 (1968) 10-5.

33 PAUWELS, L., Contribution à l'étude de l'électrodéposition du cuivre Ph.D. Thesis, Université Catholique de Louvain, Louvain (1966).

34 FISCHER, H., In Proc. 8th Interfinish Congr. Zurich - Forster Verlag, 1973 p 50.

35 EVENEPOEL, J. Cl., and WINAND, R., Etude des possibilités d'obtention de dépôts cohérents d'argent par électrolyse de solutions de nitrates - lère partie - Influence des facteurs habituels de l'électrolyse, Revue ATB Mét., 10 (1970) 133-146.

36 LAMBERT, M., and WINAND, R., Electrocristallisation du cuivre à densité de courant élevée, Oberfläche /Surface, 18 (1977) 208-216. 
37 DEGREZ, M., and WINAND, R.,

Détermination des paramètres cinétiques de l'électrodéposition du cuivre à haute densité de courant. Cas de solutions sulfuriques sans inhibiteur, Electrochim. Acta, 29 (1984) 365-372.

38 ANDRIANNE, P.A., DUBOIS, J.P., and WINAND, R., Electrocrystallization of copper in chloride aqueous solutions, Met. Trans. AIME, 8 B (1977) 315-321.

39 ALBERT, L., and WINAND, R., Copper electrowinning in chloride aqueous solutions, 111 th AIME Annual Meeting Dallas Feb. 1982, TMS Paper Selection no. A 82-26.

40 SCOYER, J., Contribution à l'étude de l'électrocristallisation du cobalt en solutions de chlorures, Ph.D. Thesis, Université Libre de Bruxelles (1977).

41 SCOYER, J., and WINAND, R., Electrocrystallization of cobalt from acid chloride solution, Surface Technology, 5 (1977) 169204.

42 DEGREZ, M., HUBEAU, V., and WINAND, R., Further progress in bright cobalt electroplating in chloride solutions without levelling agent, Rev. ATB Met., 22 (1982) 157-159.

43 DESPIC, A.R., and POPOV, K.I., Transport-controlled of metals, in : CONWAY, B.E., and BOCKRIS, J.O'M. (Eds.), Modern Aspects of electrochemistry, n 7, Plenum Press, New York, NY, 1972, pp. 199-313.

44 WINAND, R., and HARLET, Ph., High current density copper electrorefining, in : HOFFMANN, J.E., BAUTISTA, R.G., ETTEL, V.A., KUDRYK, V., and WESELY, R.J., (Eds.), The electrorefining and mining of copper, TMS Conf. Proc., Denver, CO, 1987, pp. 239-267.

45 WEYMEERSCH, A., WINAND, R., and RENARD, L., Zinc electrodeposition at high current denisty, Part 1 - Electrochemical study, Plating and Surface finishing, 68 (1981) 56-59.

46 WEYMEERSCH, A., WINAND, R., and RENARD, L., Zinc electrodeposition at high current density, Part 2 - Characteristics of deposits, ibid, 68 (1981) 118-120.

47 WINAND, R., US Patent 4, 304, 653, 1981.

48 WINAND, R., Transfert de matière et électrocristallization - Application à la galvanoplastie à haute densité de courant, Oberfläche/Surface, 25, (11) (1984) 369-375.

49 WEYMEERSCH, A., RENARD, L., CONREUR, J.J., WINAND, R., JORDA, M., and PELLET, C.,

High current density electroplating of zinc-nickel and zinc-iron alloys, Plating and Surface Finishing, 73 (1986) 68-73.

50 RODRIGUEZ FAJARDO, A., DEGREZ, M., and WINAND, R., Zn-Fe alloys electrodeposition at high current density, Part 1 - Current efficiency and deposit characterization, Oberfläche/Surface, 30, 8 (1989) 20-28.

51 RODRIGUEZ FAJARDO, A., DEGREZ, M., and WINAND, R., $\mathrm{Zn}-\mathrm{Fe}$ alloys electrodeposition at high current density, Part 2 - Mass transfer, $\mathrm{pH}$ at the cathode and fundamentals, ibid, 30, (9) (1989) 14-20.

52 VEREECKEN, J, and WINAND, R., Etude des possibilités d'obtention de dépôts cohérents d'argent par électrolyse de solutions de nitrates, 2d partie, Effet des inhibiteurs, Revue ATB Met., 10 (1970) 147-150.

53 VEREECKEN, J., and WINAND, R., Influence of inhibitors on the structure of silver deposits obtained by electrolysis of aqueous nitrate solutions, J. Electrochem. Soc., 123 (1976) 643-646. 
WINAND, R.,

Contribution to chloride hydrometallurgy in : KUDRYK, V., and RAO, Y.K., (Eds.), Physical Chemistry of extractive metallurgy, TMS Conf. Proc. New York (1985) pp. 209-229.

55 VEREECKEN, J., and WINAND, R.,

Influence of polyacrylamides on the quality of copper deposits from acidic copper sulphate solutions, Surface Technology, 4 (1976) 227-235.

56 WINAND, R., and FONTANA, A., A strategy to improve the quality of SX electrowon copper, Trans. IMM, Sect. C, 92 (1983) c2737.

57 BOCKRIS, J.O'M., and RAZUMNEY, G., Fundamental Aspects of Electrocrystallization, Plenum Press, New York, NY, 1967, Chapter II.

58 TROCH, G., Influence d'inhibiteurs organiques sur l'électrocristallisation du cuivre, Ph.D. Thesis, Université Libre de Bruxelles, Bruxelles (1983).

59 TROCH, G., DEGREZ, M., and WINAND, R., Influence of cysteine, tyrosine and gelatin on copper electrodeposition in sulphuric solution, Extended Abstracts, Spring Meeting Electrochem. Soc., Cincinnati, Ohio, (1984) 406-407.

60 TSHULA KABONGO, Contribution à l'étude de l'êlectroraffinage du cuivre, Ph.D. Thesis, Université Libre de Bruxelles, Bruxelles (1983).

61 WINAND, R., TROCH, G., DEGREZ, M., and HARLET, Ph., Industrial electrodeposition of copper. Problems connected to the behaviour of organic additions in WARREN I.H. (Ed.) Application of Polarization Measurements in the Control of Metal Deposition, Process Metallurgy, Elsevier, Amsterdam, 1984, pp. 133-145.

62 DEGREZ, M., DELPLANCKE, J.L., and WINAND, R., Simulation of hydrodynamics and inhibitor consumption in hydrometallurgical plants, $J$. Appl. Electrochem., 20 (1990) 110-115.

63 WINAND R., DEGREZ, M. and BASTIN, V., Copper electrocrystallization and the continuous monitoring of electrorefining additives, in : COOPER, W.C., KEMP, D.J., LAGOS, G.E., and TAN, K.O., (Eds.), Hydrometallurgy and Electrometallurgy of copper, Copper 91, vol. III, Pergamon Press, New York, NY, 1991, pp. 341-354.

64 DELPLANCKE, J.L., ONGARO, M., and WINAND, R., Growth of electrodeposited copper on anodized titanium, submitted for publication in the Journal of Applied Electrochemistry.

65 DELPLANCKE, J.L., SUN, M., O'KEEFE, T.J., and WINAND, R., Nucleation of electrodeposited copper on anodized titanium, Hydromet., 23 (1989) 47-66.

66 THIRSK, H.R., and HARRISON, J.A., A Guide to the Study of Electrode Kinetics, Academic Press, New York, NY,1972, pp. 115-135.

67 DELPLANCKE J.L., SUN M., O'KEEFE T.]. and WINAND R; Production of thin copper foils on microporous titanium oxide substrates, Hydrometallurgy, 24 (1990) 179-187.

68 VANDEN BRANDE, $P$., Contribution à l'étude de la nucléation et de la croissance des dépôts de cuivre par pulvérisation cathodique magnétron et par électrolyse.

Ph.D. Thesis, Université Libre de Bruxelles, Bruxelles (1990).

69 VANDEN BRANDE, P., and WINAND, R., Nucleation and initial growth of copper electrodeposits under galvanostatic conditions, to appear in Surface and Coatings Technology. 


\begin{tabular}{|c|c|c|c|c|c|c|c|}
\hline & \multicolumn{6}{|c|}{$\mathrm{J} / \mathrm{C}_{\mathrm{M}} \mathrm{e}_{+}$} \\
\hline & & $\begin{array}{l}\text { Very } \\
\text { low }\end{array}$ & Low & Medium & High & $\begin{array}{l}\text { Very } \\
\text { high }\end{array}$ & \\
\hline \multirow{5}{*}{ 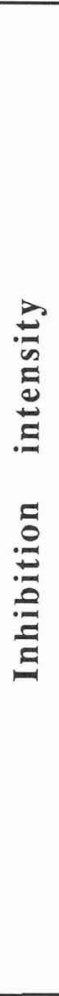 } & $\begin{array}{l}\text { Very } \\
\text { low }\end{array}$ & $\begin{array}{l}\text { No deposit } \\
\text { or FI or screw } \\
\text { dislo- } \\
\text { cation } \\
\text { No nuc- } \\
\text { leation }\end{array}$ & $\sum_{\text {incre }}$ & $\left.\right|_{N, 2} ^{A}$ & FI dendrites & 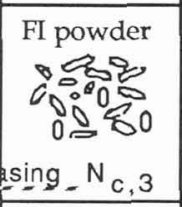 & \\
\hline & Low & 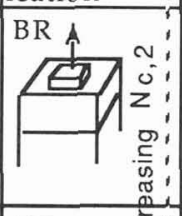 & increasing & $\mathrm{N}, \mathrm{c}, 2$ & ${ }_{n}^{\mathrm{FI}}$ & $\begin{array}{l}\text { FI powder } \\
\text { or } \\
\text { UD if bad } \\
\text { crystal- } \\
\text { lization } \\
\text { ling } N_{C, 3}\end{array}$ & $\begin{array}{l}\text { hydrogen } \\
\text { evolution } \\
\text { or }\end{array}$ \\
\hline & Medium & 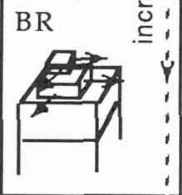 & BR & 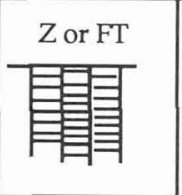 & $\frac{\mathrm{FT}}{\mathrm{E}}$ & 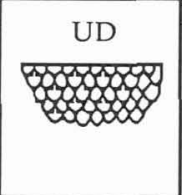 & $\begin{array}{l}\text { discharge } \\
\text { of } \\
\text { another } \\
\text { ion }\end{array}$ \\
\hline & High & $\frac{z}{N}$ & 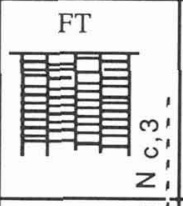 & 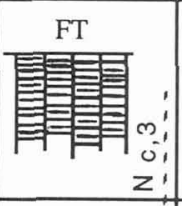 & $\begin{array}{l}\text { UD } \\
\text { w }\end{array}$ & 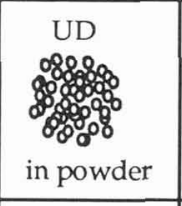 & \\
\hline & $\begin{array}{l}\text { Very } \\
\text { high }\end{array}$ & 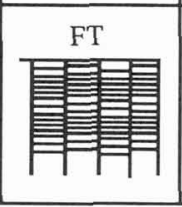 & 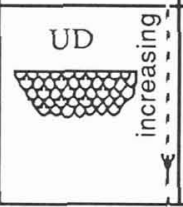 & 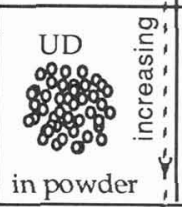 & $\begin{array}{l}\text { hydro } \\
\text { discha }\end{array}$ & $\begin{array}{l}\text { fen evoluti } \\
\text { or } \\
\text { ge of anoth }\end{array}$ & er ion \\
\hline
\end{tabular}

Figure 1 - Diagramme détaillé montrant les modes de croissance des dépôts électrolytiques polycristallins et les types de dépôts résultants, en fonction de $\mathrm{J} / \mathrm{C}_{\mathrm{Me}} \mathrm{Z}^{+}$et de l'intensité de l'inhibition. 


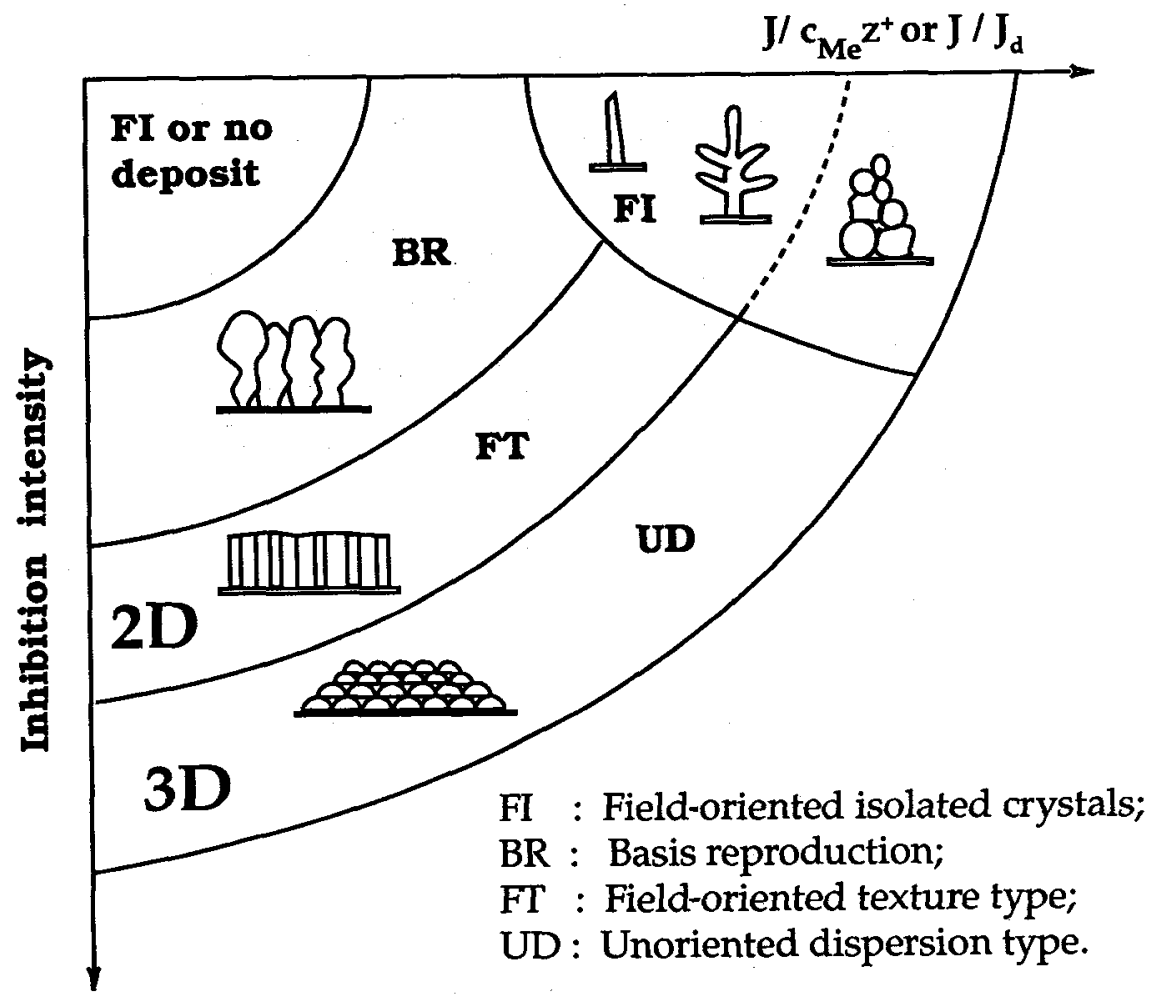

In abscissa : mass transfer characterized by $\mathrm{J} / \mathrm{c}_{\mathrm{Me}} \mathrm{Z}+$ or $\mathrm{J} / \mathrm{J}_{\mathrm{d}}$

$\mathrm{J}: \quad$ current density

$\mathrm{C}_{\mathrm{Me}} \mathrm{Z}+$ : bulk concentration of metal ion to be discharged

$\mathrm{J}_{\mathrm{d}}:$ diffusion limiting current density

In ordinate: inhibition intensity

2D : bidimensional nucleation;

3D : tridimensional nucleation.

Figure 2 - Diagramme simplifié montrant les domaines de stabilité des types de dépôts polycristallins en fonction de $\mathrm{J} / \mathrm{C}_{\mathrm{Me}^{+}}\left(\mathrm{ou} \mathrm{J} / \mathrm{J}_{\mathrm{d}}\right)$ et de l'intensité de l'inhibition. 


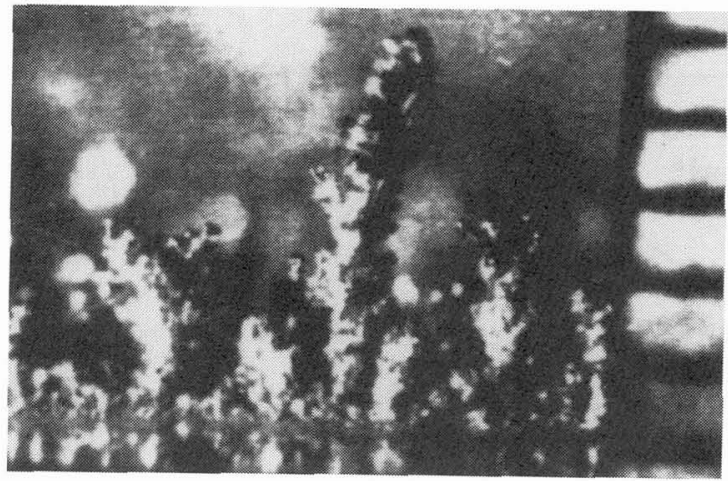

Figure 3 - Dépôt d'argent en solution de nitrates. Mince couche BR sur le substrat suivie de grandes dendrites FI. La distance entre deux lignes sur l'échelle est de $1 \mathrm{~mm}$ (de réf. [35])

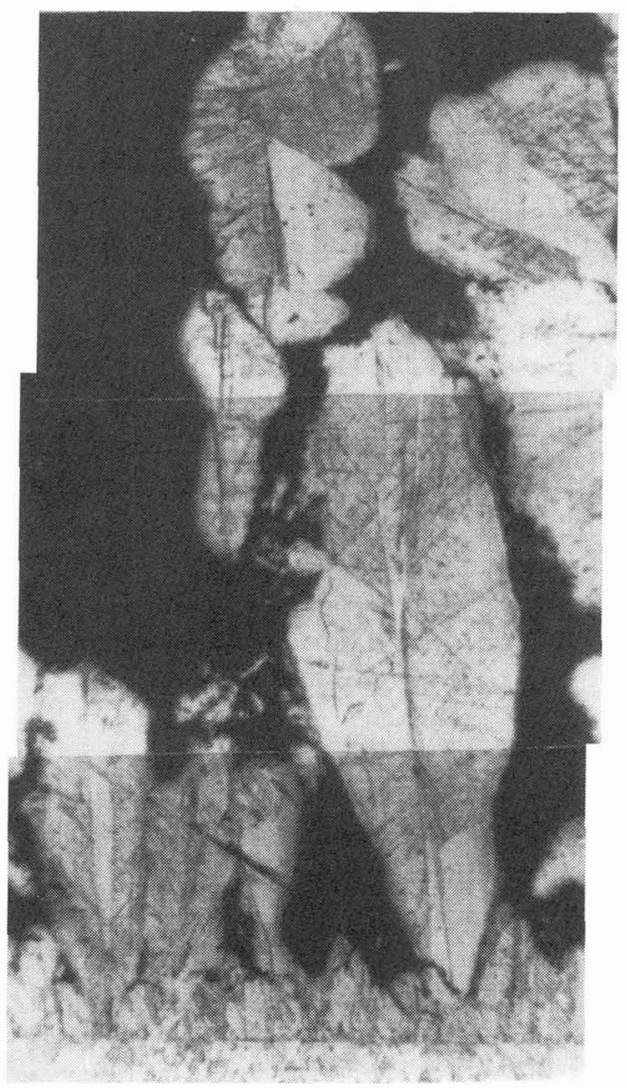

Figure 4 - Coupe métallographique dans un dépôt de cuivre obtenu d'une solution contenant $0,7 \mathrm{M} \mathrm{CuCl}, 4 \mathrm{M} \mathrm{NaCl}, 0,5 \mathrm{M} \mathrm{HCl} ; 30^{\circ} \mathrm{C} ; 24 \mathrm{~h}$; $2,5 \mathrm{~A} \mathrm{dm}^{-2}$. Mince couche initiale $\mathrm{BR}$ suivie de cristaux FI peut-être partiellement maclés (de réf. [38]) 


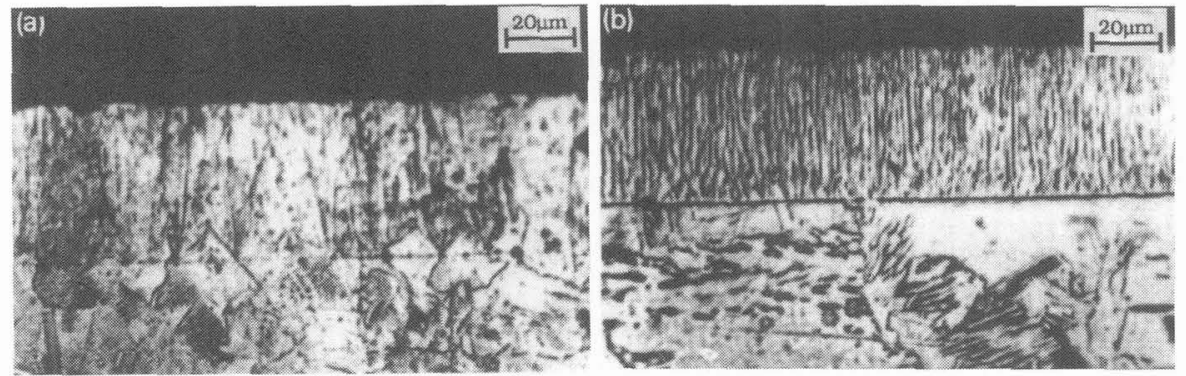

Figure 5 - Types BR et FT observés pour du cobalt déposé au départ d'une solution de chlorures à $\mathrm{pH} 4-4,5$ (de réf. [41])

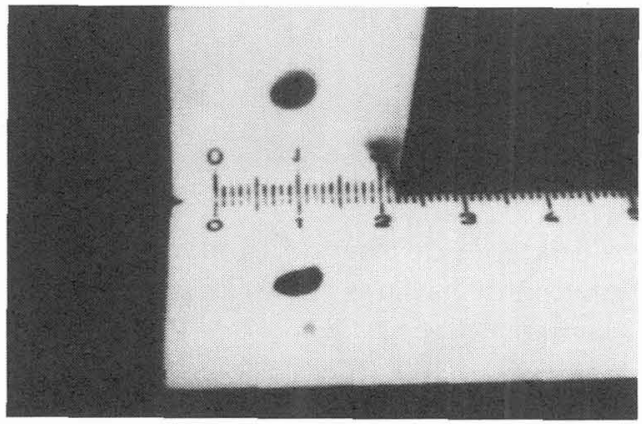

Figure 6 - Pouvoir réfléchissant d'un dépôt de cobalt brillant. Solution $1 \mathrm{M} \mathrm{CoCl}_{2}, 1 \mathrm{M} \mathrm{NaCl} ; 25^{\circ} \mathrm{C} ; 32 \mathrm{~A} \mathrm{dm}^{-2}$. Dépôt de cobalt de 3 micromètres d'épaisseur sur un dépôt de cuivre de 3 micromètres. Substrat en acier doux (de réf. [42]) 

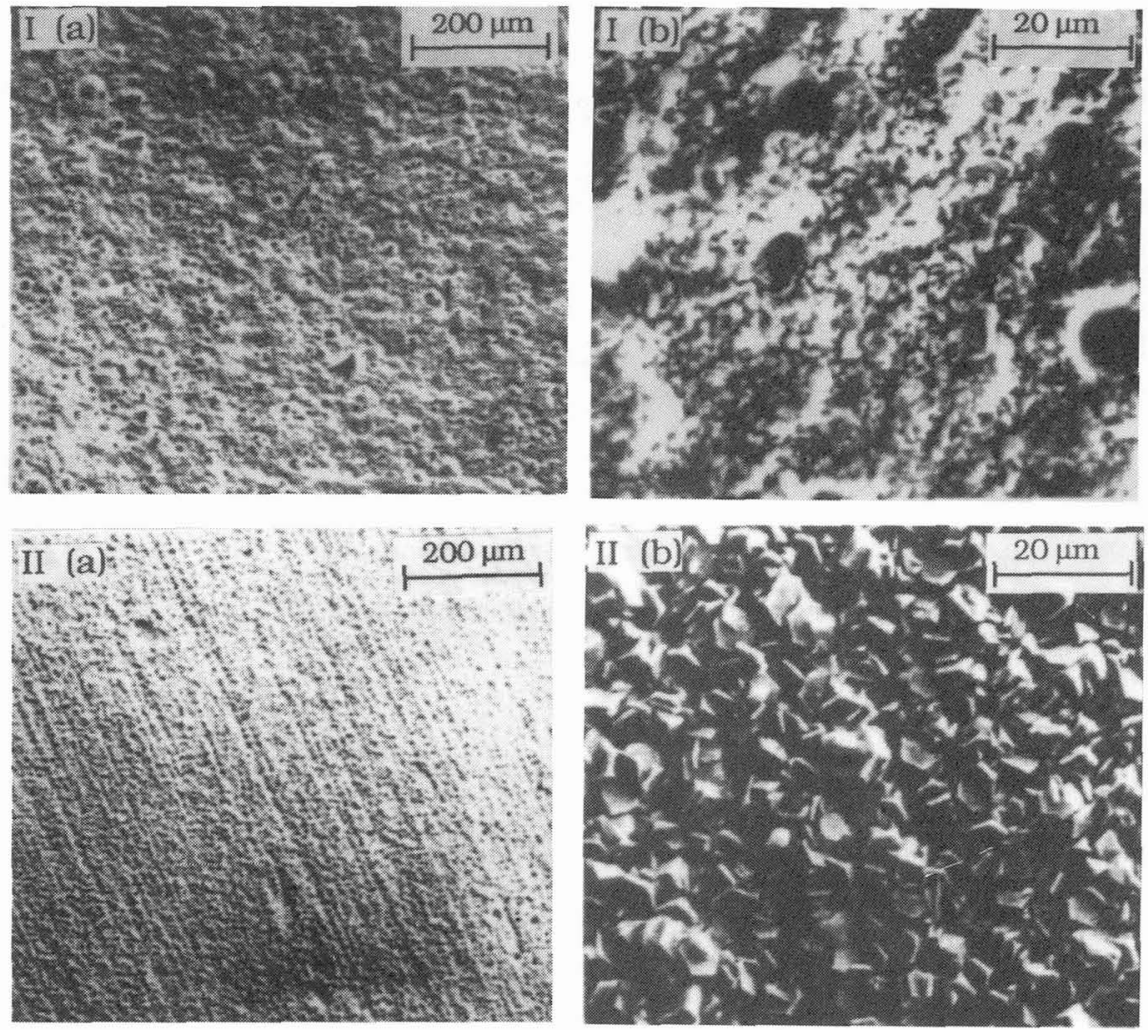

Figure 7 - Surface d'un dépôt de zinc de 10 micromètres d'épaisseur sur acier doux. Solution de sulfates : $80 \mathrm{~g} / 1 \mathrm{Zn}^{2+}, 135 \mathrm{~g} / 1 \mathrm{H}_{2} \mathrm{SO}_{4} ; 50^{\circ} \mathrm{C}$; vitesse d'écoulement : $3 \mathrm{~m} \mathrm{~s}^{-1}$

I : $10 \mathrm{~A} \mathrm{dm}^{-2}$ (a) $\times 100$, (b) $\times 1000$

II : $200 \mathrm{~A} \mathrm{dm}^{-2}$ (a) $\times 100$; (b) $\times 1000$

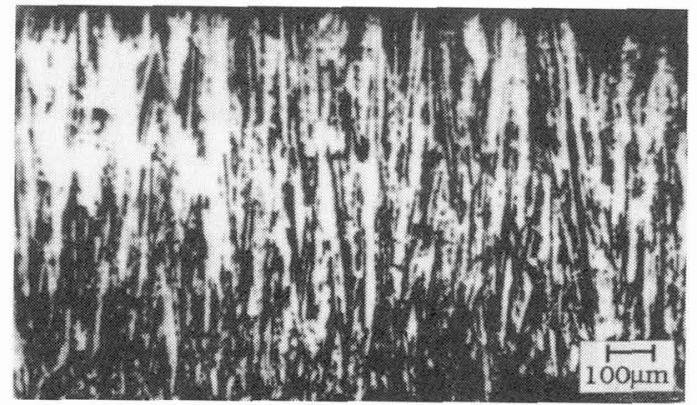

Figure 8 - Coupe métallographique d'une cathode de cuivre obtenue au laboratoire dans des conditions d'électroextraction. Electrolyte Gecamines avec $4 \mathrm{mg} / 1$ Jaguar; $55^{\circ} \mathrm{C} ; 2,4 \mathrm{~A} \mathrm{dm}^{-2} ; 48 \mathrm{~h}$. Type FT (de réf. [56]) 

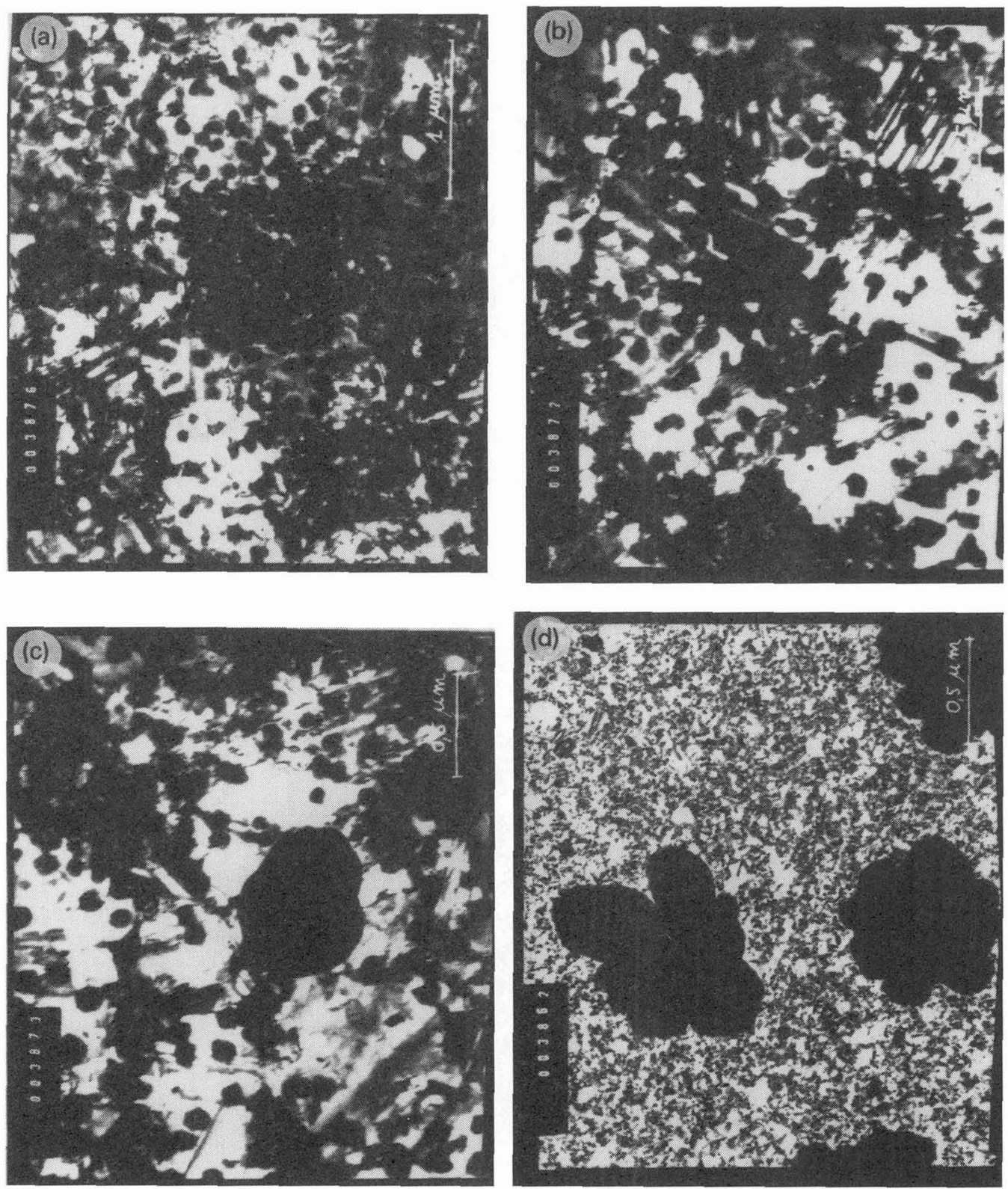

Figure 9 - Phases initiales de germination et croissance d'un dépôt de cuivre sur carbone amorphe. Solution à $0,5 \mathrm{M} \mathrm{CuSO}_{4} ; 20 \mathrm{~g} / 1 \mathrm{H}_{2} \mathrm{SO}_{4}$; désaérée; $25^{\circ} \mathrm{C} ; 3 \mathrm{~A} \mathrm{dm}^{-2}$. Quantités d'électricité : (a) $0,6 \mathrm{C} \mathrm{dm}^{-2}$; (b) $1 \mathrm{C} \mathrm{dm}^{-2}$; (c) $5 \mathrm{C} \mathrm{dm}^{-2}$; (d) $9 \mathrm{C} \mathrm{dm}^{-2}$ 